American Journal of

Food Sciences and Nutrition

(AJFSN)

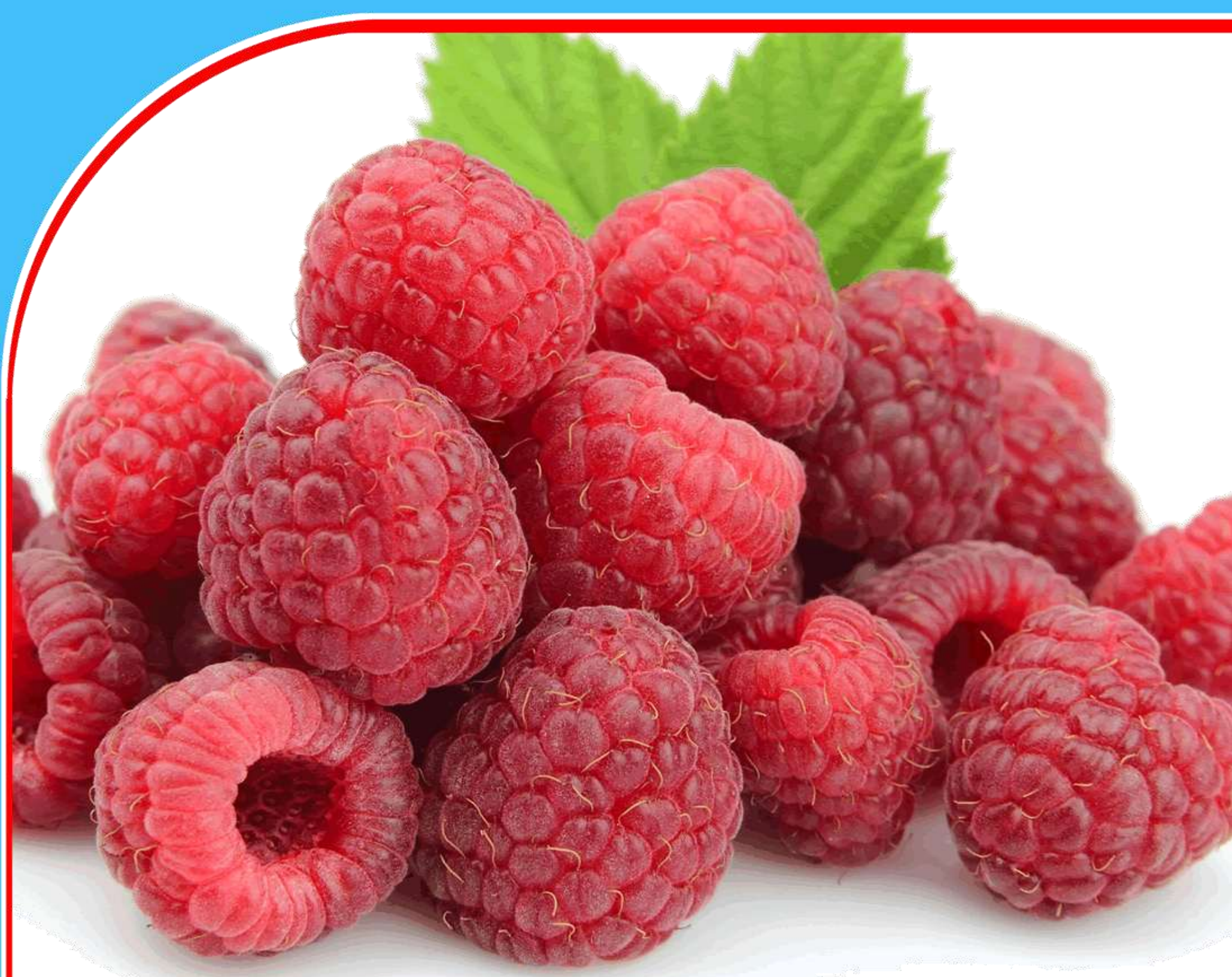

Raisins with Clarified Butter or Ghee for The Relief Of Acute And Chronic Constipation - A Clinical Study

Dr. Swati Suresh Khartode, Dr. Kiran Shinde, Dr.

Chandrakant Sahare, Dr. Sushant Shinde, Dr. Chagan Khartode, and Dr. Namdev Jagtap.

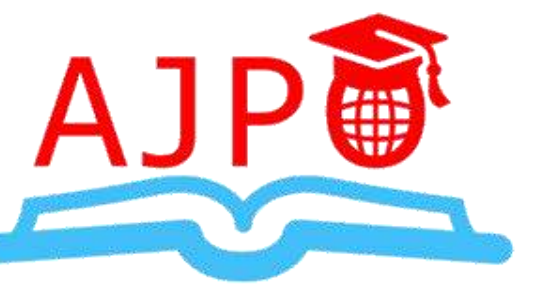




\title{
Raisins with clarified butter or ghee for the relief of acute and chronic constipation - A Clinical Study
}

\author{
Dr. Swati Suresh Khartode ${ }^{1}$, Dr. Kiran Shinde ${ }^{2}$,Dr. Chandrakant Sahare ${ }^{3}$, Dr. Sushant \\ Shinde $^{4}$, Dr. Chagan Khartode ${ }^{5}$, Dr. Namdev Jagtap ${ }^{6}$. \\ ${ }^{1}$ BAMS, MSC- Dietetics and Food Service Management, Consultant Dietitian, Vishwaraj \\ Hospital, Pune, India \\ ${ }^{2}$ MBBS, MD-General Medicine, DM-Gastroenterology. Consultant Gastro-enterologist, \\ Vishwaraj Hospital, Pune, India \\ ${ }^{3}$ MBBS DCH DNB PGPN, Head of the pediatric department, Vishwaraj Hospital, Pune, India \\ ${ }^{4} \mathrm{MBBS}$, DNB- Medicine, Consultant Physician, HOD of casualty department, Vishwaraj \\ Hospital, Pune, India \\ ${ }^{5}$ MBBS, DNB- Medicine, Consultant Physician, Vishwaraj Hospital, Pune, India \\ ${ }^{6}$ MBBS, MD-General Medicine, Consultant Physician, Vishwaraj Hospital, Pune, India \\ Corresponding Author's Email: khartode.swati@gmail.com
}

\begin{abstract}
Background: Constipation is a very distressful symptom, affecting to all age group. The only treatment is laxatives, which is having many adverse effects mainly dependency on laxatives.

Purpose: To evaluate the laxative effect of raisins and to get relief from daily dependency on laxative medicines for acute and chronic constipation

Methods: Interventional / Experimental study of raisins and clarified butter or ghee performed at 'Vishwaraj Hospital' Pune, Maharashtra, India from March 2021 to June 2021, after appropriate ethical approval obtained from the Vishwaraj Hospital's Ethics Committee (Registration numberECR/1138/Inst/MH/2018). Fifty one patients of constipation who were on laxatives or PR enema enrolled in this study, diagnosis confirmed by gastro-enetrologist. Laxatives had been asked to stop before enrollment. Questionnaire form were given at the time of enrollment and telephonic follow up for OPD patients and by visiting to admit patients had been taken on $2^{\text {nd }}$ day after consumption of raisins and clarified butter or ghee then on $7^{\text {th }}$ and $15^{\text {th }}$ day. Forms were collected once they were visit to their respective doctors for follow up.

Results: Statistical tool used in this study is the $95 \%$ confidence interval. The variables of interest were 1) percent of patients have restarted laxatives or not and 2) number of days required to get relief from constipation. $94.12 \%$ of patients $(n=48)$ did not restart laxatives after consumption of raisins and clarified butter or ghee and the population percent of patients that might not restart laxatives would be $88 \%$ to $100 \%$ with $95 \%$ confidence. Also the number of days to get relief from constipation after consumption of raisins and clarified butter or ghee for a patient in population would be 3-4 days with $95 \%$ confidence.

Conclusion: Raisins and $\mathrm{CB}$ or ghee had given relief from acute and chronic constipation; worked very well on irritable bowel syndrome, fissure in ano, hemorrhoids and helped to stop PR bleeding and bloating causes by these diseases. Patients got relief from constipation on 3rd and 4th day. This study concluded that raisins with clarified butter or ghee can be taken instead of laxatives or enema to get relief from constipation; this might be the great substitute for laxatives and per rectum enema.
\end{abstract}

Key words: Constipation, Clarified butter, Enema, Ghee, Laxatives, Raisins 
American Journal of Food Sciences and Nutrition

ISSN 2789-3154 (Online)

Vol.3, Issue 2, pp 1- 15, 2021

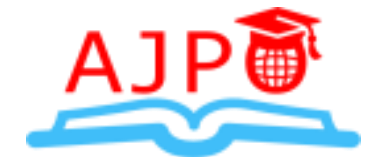

www.ajpojournals.org

\section{INTRODUCTION}

Constipation is a syndrome that is defined by bowel symptoms such as difficult or infrequent passage of stool, hardness of stool, or a feeling of incomplete evacuation that may occur either in isolation or secondary to another underlying disorder (Bharucha, 2013). Constipation is defined differently by different people. Most often the condition is infrequency of evacuation, which is less than three bowel movements per week in normal individuals. For other patients, abnormally hard stools or defecation that requires excessive straining defines constipation. (Schiller, 2001).

In 2004, The American College of Gastroenterology defined constipation based upon symptoms including unsatisfactory defecation with either infrequent stools, difficult stool passage or both. Any recent change in bowel habits, if persistent, may be cause for concern. Thus there is currently no ideal definition for constipation; history and physical examination can be considered the main initial approaches. Many definitions are described by using a self-reported constipation and the formal criteria. Many definitions of chronic constipation are related to scientific considerations such as secondary causes (medications), neurological, or systemic diseases. However, it is considered primary or idiopathic. Constipation among older people is far more common than younger people. Common causes of constipation in the elderly are linked to several factors including lack of normal bowel movements or aging, lack of proper diet, lack of adequate fluid intake, lack of adequate physical activity, illness or the use of drugs (Forootan, 2018).

Constipation is not a disease, but may be a symptom of another medical problem. Constipation may last for a short or long time i.e. acute or chronic. As per the National Institute of Diabetes and Digestive and Kidney Diseases (NIDDK), Symptomatic presentation of constipation is 1. Difficulty passing stool, 2. Straining when passing stool, 3. Passing less stool than usual. 4. Lumpy, dry, or hard stool. Other symptoms include: Pain and cramping in the abdomen, Feeling bloated, Nausea, A loss of appetite. According to Stanford health care in 2017, the most common complications associated with constipation are discomfort and irritation that can lead to: Hemorrhoids, Rectal bleeding, Anal fissures (tears in skin around the anus), Sometimes, the difficulty passing a bowel movement can cause more serious complications, such as: rectal prolapse (the large intestine detaches inside the body and pushes out of the rectum), fecal impaction (hard, dry stool is stuck in the body and unable to be expelled naturally).

Abbott, a healthcare company, conducted a nationwide gut health survey in 2015 to assess the condition of constipation sufferers in the country, as well as the association of constipation with comorbidities such as diabetes, hypothyroidism, and anorectal disorders. The findings suggest that $22 \%$ of the adult Indian population is suffering from the condition, with $13 \%$ complaining of severe constipation. $6 \%$ of the Indian population suffers from constipation associated with certain comorbidities. This survey states higher prevalence of constipation in metro cities (Abbott India, 2015)

Various metabolic disorders like diabetes, hypothyroidism is likely to cause constipation. Also, ano-rectal disorders and pregnancy in women sometimes causes constipation. Constipation tends to be more severe when associated with some of these comorbidities. There are essentially two types of treatment for constipation after efforts have been made to remove the underlying cause that is oral laxatives and suppositories or enemas. Oral laxatives can be broadly divided into 
bulking agents, osmotic laxatives, stimulant laxatives, and stool softeners. The choice of laxative is largely a matter of personal preference and availability with few published recommendations (Vincent \& Preiser, 2015). Laxatives can help with bowel movements, but regular use of certain laxatives can be habit forming especially stimulant laxatives. This means that the more a person depends on laxatives, the greater their risk of constipation when they stop using them. Overuse of laxatives can also lead to dehydration, an electrolyte imbalance (Felman, 2019). Also constipation and laxative use increases the risk of colon cancer (Watanabe, 2004).

So it is better to adopt healthy lifestyle habits instead of taking laxatives for the relief of constipation. Increase intake of fibers and moderate activity proven to avoid constipation. Raisins are rich in soluble and insoluble fibers (Camire \& Dougherty, 2003). Raisins are commonly recommended as good food choices because of their nutrient content and their health- promoting benefits. They are rich sources of potassium and dietary fiber and provide an array of phytonutrients (Karadeniz et al., 2000).

Raisins are excellent sources of insoluble and soluble fibers and also provide significant amounts of fructooligosaccharides (FOS) including inulin. A 1- oz serving (28 gm) of raisins would provide $3.0 \mathrm{~g}$ of dietary fiber per 90 calories giving them an excellent "source of dietary fiber" rating. Raisins are rich in FOS, grapes have a very low FOS content (Camire \& Dougherty, 2003). This inulin has a very important prebiotic effect in addition to other health benefits for humans (Anderson \& Waters, 2013). Prebiotics are a category of nutritional compounds grouped together by the ability to promote the growth of specific beneficial (probiotic) gut bacteria (Kelly, 2008). The prebiotic effect of inulin-type fructans has been extensively confirmed. The usual target microorganisms are bifidobacteria, with major increases in their numbers occurring on ingestion. Often, 0.5-1.0 $\log 10$ numerical increases are seen. This constitutes a major shift in the gut micro biota toward a "healthier" composition (Kolida \& Gibson, 2007).

Because of their rich content of dietary fiber, potassium, and phytonutrients, the regular consumption of raisins has the potential to lower blood pressure. Raisin intake decreases fasting serum LDL (low density lipoprotein) cholesterol, triglycerides, and oxidized LDL cholesterol, also regular consumption increases serum antioxidant capacity (Anderson et al., 2013). Raisins are a low to moderate glycemic index (GI), with a correspondingly low insulin index (II). The study conducted by Kim, et al., (2008) shows that raisins are a low to moderate glycemic index (GI), with a correspondingly low insulin index (II). The study subjects consisted 10 healthy sedentary, 11 aerobically trained adults, and 10 pre diabetic adults, Subjects consumed $50 \mathrm{~g}$ of available carbohydrate from raisins and from a glucose solution (reference food) on 2 separate occasions. Serum glucose and insulin concentrations were measured from blood samples at baseline and at 15, 30, 45, 60, 90, and 120 minutes (and 150 and 180 minutes for pre diabetic group) post prandial. The GI of raisins was low (GI, $\leq 55)$ in the Sedentary $(49.4 \pm 7.4)$ and Pre diabetic (49.6 \pm 4.8$)$ groups and was moderate (GI, 55-69) in the aerobically trained group (62.3 $\pm 10.5)$.

Grapes and raisins provide phenolic antioxidants, which contribute to their potential health benefits. Golden raisins had the highest antioxidant capacity and phenolic content (Parker et al., 2007) 
Ghee is a variation of clarified butter that is popular in the culinary traditions of the Middle East and India. It is made from cow milk butter, which is treated with low heat until the water evaporates, leaving behind milk solids. The solids are skimmed off or strained if needed. What remaining part is the clarified liquid fat known as ghee? Because ghee is treated with low heat, usually less than 100 degrees, it retains more nutrients than standard clarified butter. (Ghee: Is It Good for You? 2020). Ghee has been utilized for thousands of years in Ayurveda as a therapeutic agent. In ancient India, ghee was the preferred cooking oil. Ghee increases the digestive fire (agni) and improves absorption and assimilation. It improves memory and strengthens the brain and nervous system. It lubricates the connective tissues, thereby rendering the body more flexible (Sharma et al., 2010).

The incorporation of cow ghee in the dosage form is able to enhance the absorption of drug. Increase in the fluidity of the membrane facilitates the trans-cellular absorption of the drug. Pure Ghee is rich in antioxidants which improves the digestibility of proteins by $36 \%$ and increases bio availability by $62 \%$ when it is added to vitamin A deficient diet. Ghee also aids in absorption of minerals from other foods, increasing the retention of calcium up to $45 \%$ and phosphorous up to $57 \%$. It is a safe alternative for people who are lactose intolerant since all the milk proteins have been removed during the clarifying process (Kiran et al., 2015). Also the presence of fat in a meal, with which a vitamin D-3 supplement is taken, significantly enhances absorption of the supplement (Dawson-Hughes et al., 2015).

Ghee improves memory and strengthens the brain and nervous system. Ghee is heavily utilized in Ayurveda for numerous medical applications, including the treatment of allergy, skin, and respiratory diseases. Ghee carries the therapeutic properties of herbs to all the body's tissues. It is an excellent anupana (vehicle) for transporting herbs to the deeper tissue layers of the body. (Lad, 1999). Proper digestion, absorption, and delivery to a target organ system are crucial in obtaining the maximum benefit from any therapeutic formulation; the lipophilic action of ghee facilitates transportation to a target organ and final delivery inside the cell since the cell membrane also contains lipid. (Sharma, 1990).

In India, partially hydrogenated vegetable oil known as vanaspati was introduced in the 1960s and promoted as "vegetable ghee." It contains up to $40 \%$ trans fatty acids. (Ghafoorunissa, 2008). Singh et al., (1996) studied the association of ghee and vegetable intake with higher risk of coronary artery disease (CAD) in rural and urban populations in northern India. Increased prevalence of CAD was associated with the intake of ghee plus vegetable ghee, but the risk was lower with consumption of ghee alone. A study on a rural population in Rajasthan, India, revealed a significantly lower prevalence of CAD in men who consumed higher amounts of ghee (Gupta, 1997)

Ghee can be served to the people of all age groups for their nourishment. It is a good carrier of fat-soluble vitamins (A, D, E and $\mathrm{K}$ ) along with essential fatty acids (linolenic and arachidonic acid) which are responsible for wellbeing. (Kumar et al., 2018). Short chain fatty acids in ghee give a longer storage life than butter with less rancid off-flavor. These fatty acids are potentially beneficial for reducing body weight and body fat. Further, these fatty acids are easily digestible 
and transferred directly from the intestine to the portal circulation and are a preferred source of energy ( $\beta$-oxidation) (Kwak, et al., 2013).

Thus, raisins and clarified butter (short form CB will be used for clarified butter in this article) or ghee have multiple health benefits and rich in nutritional composition. Constipation, being one of the major complicated syndrome and adverse effects seen due to the use of laxatives to treat the constipation, there are no any significant dietary recommendation for constipation other than high fiber diet at present, so to avoid the complications of constipation and laxatives with the permanent dietary solution of raisins and $\mathrm{CB}$ or ghee, this study was carried out with the following objectives,

\subsection{OBJECTIVE}

1. To evaluate the laxative effect of raisins on constipation (Acute and chronic)

2. To get relief from dependency on laxative medicines.

\section{MATERIALS AND METHODS}

\subsection{Study Design}

Interventional / Experimental study to evaluate the Laxative Effect of soaked raisins and CB or ghee in constipation patients performed at 'Vishwaraj Hospital' Pune, Maharashtra, India from March 2021 to June 2021.

\subsection{Ethical approval}

Appropriate ethical approval was obtained from the Vishwaraj Hospital Ethics Committee (Registration number- ECR/1138/Inst/MH/2018), the study protocol, Consent form, Assent form and Case record form i.e. questionnaire form was designed and approved by the Institutional Ethics Committee before the start of the study and all subjects of study group had given written, informed consent (In English, Marathi and Hindi language).

\subsection{Selection of samples}

Fifty one patients of constipation were selected by Randomization method in which, 39 patients were adult and 12 patients were pediatric patients. Diagnosis of constipation was confirmed by gastroenterologist, physicians and pediatrician of hospital.

Forty seven patients were hospitalized and 4 were OPD (outdoor patients department) patients. Patients were mostly selected from IPD (indoor patients department), admitted for other reasons who complained of constipation.

Two patients were having acute constipation and 49 patients were having chronic constipation.

Ten patients were having secondary constipation and 41 patients were having primary constipation.

Dose of raisins: 25-30 for adult and 10-20 for pediatric

Dose of CB or ghee: 2 tablespoon for adult and 1 tablespoon for pediatric

Patients were asked to eat above mentioned dose at their home after taking signature on consent form and after giving all written and verbal information about raisins and CB or ghee. 
All patients were asked to stop the laxatives whichever they had previously consuming, no any dietary changes recommended to patients.

After consuming raisins and $\mathrm{CB}$ or ghee together follow up had been taken on $2^{\text {nd }}$ day after consumption of raisins and $\mathrm{CB}$ or ghee by visiting to admit patients and by telephone once they got discharge on $7^{\text {th }}$ and $15^{\text {th }}$ day. For OPD patients follow up had been taken by telephone on $2^{\text {nd }}$, $7^{\text {th }}$ and $15^{\text {th }}$ day. Total 2 weeks follow up study. Questionnaire forms had been collected once they came for follow up with their physicians and gastroenterologist at hospital.

\subsubsection{Inclusion criteria:}

1. Confirmed symptom of constipation.

\subsubsection{Exclusion criteria:}

Patients with any of the following conditions were excluded from the study:

1. Uncontrolled Diabetic patients.

2. Constipation due to intestinal obstruction

3. Diarrhea

4. Allergic to raisins and ghee

5. Chronic Kidney disease (CKD)

\subsection{Statistical Analysis}

\section{Statistical tools used:}

There were two variables of interest in this study,

1) Success of the intervention that is proportion of patients those did not restart laxatives to get relief from constipation, after consumption of raisins and $\mathrm{CB}$ or ghee.

2) Number of days to get relief from constipation after consumption of raisins and CB or ghee

The first variable is proportion so the statistical tool used is '95\% Confidence interval for population proportion' for a 95\% confidence interval; we are $95 \%$ confident that the population proportion will lie in that obtained interval.

The second variable is continuous so the statistical tool used is '95\% Confidence interval for population mean' for a $95 \%$ confidence interval; we are $95 \%$ confident that the population mean will lie in that obtained interval.

\section{RESULTS AND DISCUSSIONS}

This clinical study performed on 51 patients of constipation in which 47 patients were hospitalized and 4 were OPD (Outdoor Patients Department) patients. Patients were mostly selected from IPD (Indoor Patients Department), admitted for other reasons who complained of constipation.

All participants were selected by randomized method with confirmed diagnosis of constipation and were using any kind of laxatives or enema to get relief from constipation. Pediatric patients 
were taking laxatives or enema whenever they have constipation, not daily. Excluded those who were having uncontrolled diabetes, chronic kidney disease, diarrhea, constipation due to intestinal obstruction and allergic to raisins and CB or ghee. All participants were asked to stop laxatives or enema whichever they were using before enrolled in this study. Also no any dietary modification has been advised to any patients. This study comprised wide range of age group from 2 years to 85 years (Table 1) with $58.8 \%$ male and $41.2 \%$ female patients (Table 2) and $76.5 \%$ adult patients with $23.5 \%$ pediatric patients. (Table 3). The detailed results with the frequency of distribution and statistical test with discussions are present below,

\subsection{Age}

One of the survey about constipation done in Chandigarh, India by Nebhinani, et al. in 2014 revealed that $83 \%$ were within the age group of $18-59$ years with mean age (years) of $38.64 \pm$ 15.57. Constipation was significantly more frequent in females than in males (20\% vs. 13\%) and in nonworking population than in working population (20\% vs. $12 \%)$.

Table 1: The frequency distribution of patients according to Age is as given below.

\begin{tabular}{|lll|}
\hline Age in years & Frequency & $\%$ \\
\hline 10 and below & 11 & 21.6 \\
11 to 20 & 4 & 7.8 \\
21 to 30 & 5 & 9.8 \\
31 to 40 & 11 & 21.6 \\
41 to 50 & 7 & 13.7 \\
51 to 60 & 5 & 9.8 \\
61 to 70 & 6 & 11.8 \\
71 to 80 & 1 & 2.0 \\
81 to 90 & 1 & 2.0 \\
Total & 51 & 100.0 \\
\hline
\end{tabular}

\subsection{Gender}

Constipation is more common in women than in men. Moreover, severe constipation is significantly seen in elderly women as compared with that of male individuals (Vazquez Roque \& Bouras, 2015).

Table 2: The frequency distribution of patients according to Gender is as given below.

\begin{tabular}{|lll|}
\hline Gender & Frequency & $\%$ \\
\hline Female & 21 & 41.2 \\
Male & 30 & 58.8 \\
Total & 51 & 100.0 \\
\hline
\end{tabular}




\subsection{Adult/ Paediatric patients}

Constipation is the most common cause of acute abdominal pain in children (Loening-Baucke \& Swidsinski, 2007). Large and hard stool, painful defecation and withholding behavior were the most frequent sign or symptoms among children with chronic functional constipation. Fresh rectal bleeding and anal fissure were the least frequent sign and symptom among children with chronic functional constipation (Dehghani, et al., 2015)

Table 3: The frequency distribution of patients according to Adult/ Paediatric patients is as given below.

\begin{tabular}{|lll|}
\hline $\begin{array}{l}\text { Adult/ Paediatric } \\
\text { patients }\end{array}$ & Frequency & $\%$ \\
\hline Adult & 39 & 76.5 \\
Paediatric & 12 & 23.5 \\
Total & 51 & 100.0 \\
\hline
\end{tabular}

\subsection{Acute/Chronic constipation}

Without early diagnosis and treatment, an acute episode of constipation can lead to anal fissure and become chronic. Early identification of constipation and effective treatment can improve outcomes for children and young people (National Collaborating Centre for Women's and Children's Health (UK). 2010). Majority patients in this study that is $96 \%(n=49)$ patients were having chronic constipation and $4 \%(\mathrm{n}=2)$ were having secondary constipation in which these two patients were suffered from neurological disorders and on nasogastric feed for long term previously as shown in table 4.

Table 4: The frequency distribution of patients according to Acute/Chronic constipation is as given below.

\begin{tabular}{|lll|}
\hline Acute/Chronic constipation & Frequency & $\%$ \\
\hline Acute & 2 & 4 \\
Chronic & 49 & 96 \\
Total & 51 & 100.0 \\
\hline
\end{tabular}

\subsection{Co-morbidity / Ano rectal disease}

Anorectal disorders like anal fissures and hemorrhoids delay the removal of stool and exacerbate chronic constipation due to pain during fecal excretion. Co-morbidity like diabetes, hypothyroidism, hyperthyroidism and psychological conditions like depression, anxiety, eating disease, also in pregnancy, menopause causes constipation and it is the most troublesome situation (Forootan et al., 2018). In this study majority of patients $67 \%(n=34)$ were not having any co-morbidity, others were having comorbidities like diabetes, hemorrhoids, fissure in ano, irritable bowel syndrome, IHD, hypertension and liver cirrhosis with ascites as shown in Table 5. 
American Journal of Food Sciences and Nutrition

ISSN 2789-3154 (Online)

Vol.3, Issue 2, pp 1- 15, 2021

www.ajpojournals.org

Table 5: The frequency distribution of patients according to Co-morbidity / Ano rectal disease is as given below.

\begin{tabular}{|lll|}
\hline $\begin{array}{l}\text { Co-morbidity /Ano rectal } \\
\text { disease }\end{array}$ & Frequency & $\%$ \\
\hline None & 34 & 66.7 \\
Bronchial asthma & 1 & 2.0 \\
COPD & 1 & 2.0 \\
Diabetes & 3 & 5.9 \\
Fissure in ano & 3 & 5.9 \\
Hemorrhoids & 2 & 3.9 \\
Hypertension & 1 & 2.0 \\
Ischemic heart Disease & 2 & 3.9 \\
Liver cirrhosis with ascites & 3 & 5.9 \\
Irritable Bowel Syndrome & 1 & 2.0 \\
Total & 51 & 100.0 \\
\hline
\end{tabular}

\subsection{Primary / Secondary Constipation}

Table 6: The frequency distribution of patients according to Primary / Secondary Constipation is as given below.

\begin{tabular}{|lll|}
\hline $\begin{array}{l}\text { Primary / Secondary } \\
\text { Constipation }\end{array}$ & Frequency & $\%$ \\
\hline Primary & 41 & 80 \\
Secondary & 10 & 20 \\
Total & 51 & 100.0 \\
\hline
\end{tabular}

In this study $80 \%(n=41)$ patients were having primary constipation and $20 \%(n=10)$ were having secondary constipation in which patients were suffered from liver cirrhosis, irritable bowel syndrome, fissure and hemorrhoids as summarized in table 6. 
3.7. On which day constipation relieved after consumption of raisins and $\mathrm{CB}$ or ghee.

Table 7: The frequency distribution of patients according to 'on which day constipation relieved after consumption of raisins and CB or ghee' along with its bar graph is as given below.

\begin{tabular}{|lll|}
\hline $\begin{array}{l}\text { On which day constipation relieved after } \\
\text { consumption of raisins and CB / ghee }\end{array}$ & Frequency & $\%$ \\
\hline No relief & 3 & 6 \\
$2^{\text {nd }}$ day & 2 & 4 \\
$3^{\text {rd }}$ day & 15 & 29 \\
$4^{\text {th }}$ day & 23 & 45 \\
$5^{\text {th }}$ day & 6 & 12 \\
$6^{\text {th }}$ day & 1 & 2.0 \\
$7^{\text {th }}$ day & 1 & 2.0 \\
Total & 51 & 100.0 \\
\hline
\end{tabular}

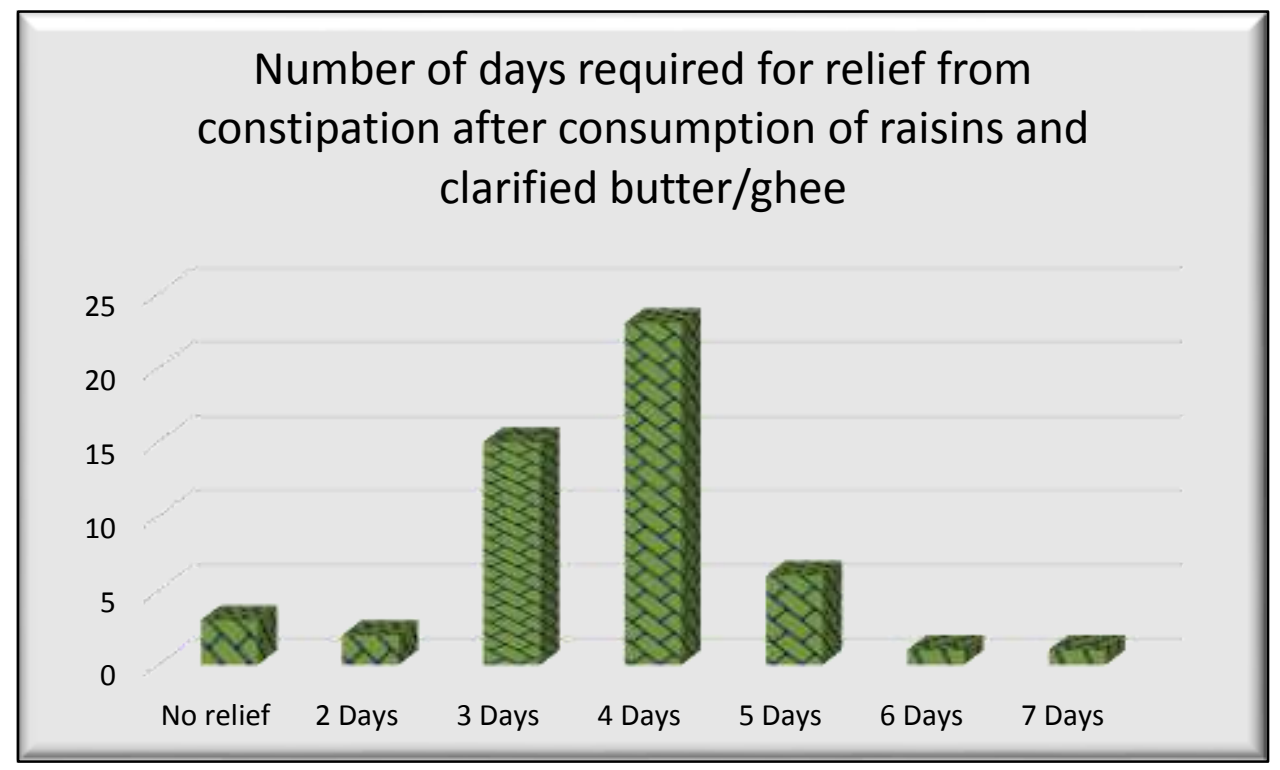

Figure 1: The frequency distribution of patients according to 'on which day constipation relieved after consumption of raisins and $C B$ or ghee

Majority of patients that is $45 \%(n=23)$ got relief from constipation on $4^{\text {th }}$ day while $29 \%(n=15)$ got relief on $3^{\text {rd }}$ day as shown in table 7 and figure 1. 


\subsection{Restarted Laxatives?}

Laxatives are among the most commonly used drugs or additives. Most are quite safe when used judiciously, intermittently when possible, and in the absence of contraindications. Bulking agents and non-absorbable compounds can cause bloating. Osmotic laxatives containing poorly absorbable ions such as magnesium or phosphate can cause metabolic disturbances, particularly in the presence of renal impairment. Senna compounds and bisacodyl are the most commonly used stimulant laxatives. Although there are data to support the neoplastic potential of this class of drugs in in vitro studies, epidemiologic data in humans so far has not established a clear link between these laxatives and colonic neoplasia. Danthron compounds should be avoided because of hepatotoxicity (Xing, Jin \& Soffer, 2001). About $94.12 \%(n=48)$ patients in this study did not restart laxatives whichever they were taking before enrollment in this study, only $5.88 \%(\mathrm{n}=3)$ patients restarted laxatives as they did not get relief from constipation. No one from pediatric group restarted laxatives or enema which they had occasionally using after consumption of raisins and CB or ghee as summarized in table 8 and figure 2.

\section{This is the major finding in this study.}

Also all patients those did not restart laxatives or enema got relief from bloating i.e. gaseous abdominal distension, PR bleeding, abdominal pain they had before.

Table 8: The frequency distribution of patients according to restarted laxatives or not, along with its bar graph is as given below.

\begin{tabular}{|lll|}
\hline Restarted Laxatives? & Frequency & $\%$ \\
\hline No & 48 & 94.12 \\
Yes & 3 & 5.88 \\
Total & 51 & 100.0 \\
\hline
\end{tabular}

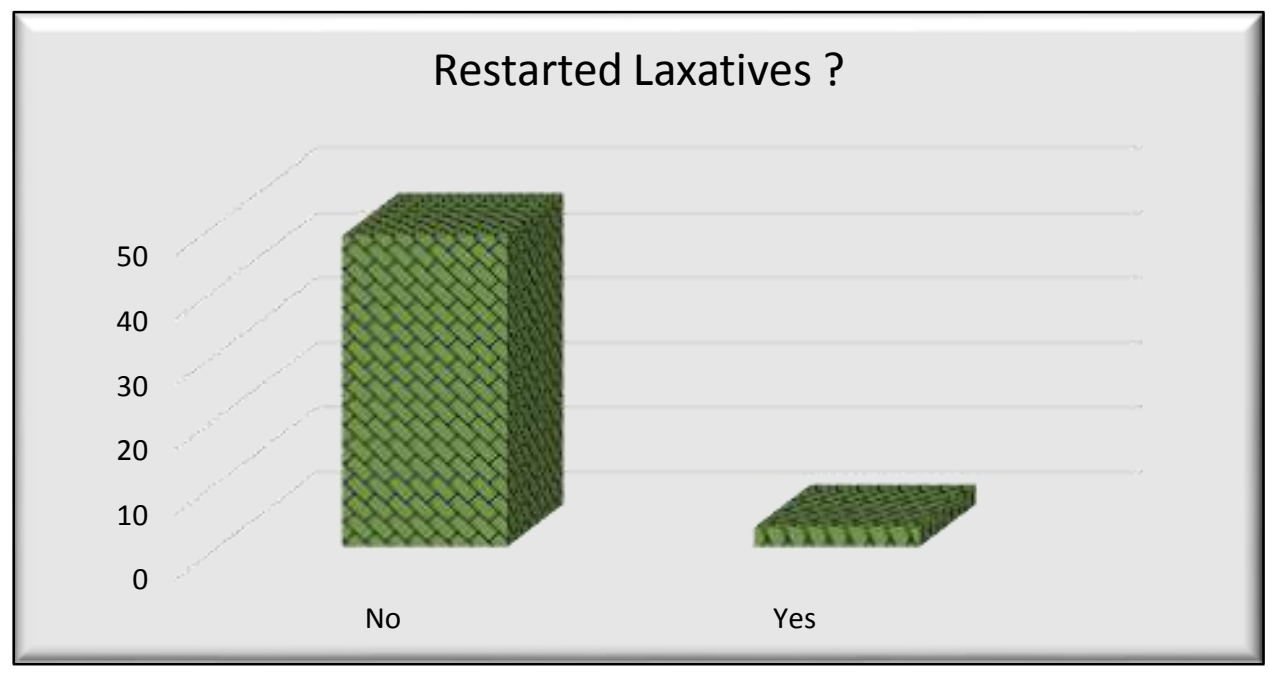

Figure 2: The frequency distribution of patients according to restarted laxatives. 
American Journal of Food Sciences and Nutrition

ISSN 2789-3154 (Online)

Vol.3, Issue 2, pp 1- 15, 2021

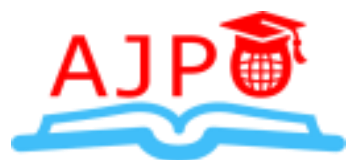

www.ajpojournals.org

\section{Variables of Study}

1) Success of the nutritional intervention that is proportion of patients those did not restart laxatives to get relief from constipation, after consumption of raisins and $\mathrm{CB}$ or ghee.

\begin{tabular}{|c|c|c|c|c|c|}
\hline \multicolumn{3}{|l|}{$\begin{array}{l}\text { Patients those did not restart laxatives to } \\
\text { get relief from constipation, after } \\
\text { consumption of raisins and CB or ghee }\end{array}$} & \multicolumn{2}{l|}{$\begin{array}{l}\text { 95\% Confidence Interval of } \\
\text { Population Percentage }\end{array}$} \\
\hline $\mathrm{N}$ & $\mathrm{n}$ & $\%$ & Proportion & Lower & Upper \\
\hline 51 & 48 & 94.12 & 0.94 & 87.66 & $100.57 \approx 100$ \\
\hline
\end{tabular}

The percentage of patients those did not restart laxatives to get relief from constipation, after consumption of raisins and CB or ghee is $94.12 \%$. The $95 \%$ confidence interval for population percent of patients those did not restart laxatives to get relief from constipation, after consumption of raisins and CB or ghee is $(87.66 \%, 100.57 \%)$ approximately $(88 \%, 100 \%)$. This implies in the population, the percent of patients those did not restart laxatives to get relief from constipation, after consumption of raisins and CB or ghee would be $88 \%$ to $100 \%$ with $95 \%$ confidence.

2) Number of days for relief from constipation after consumption of raisins and CB or ghee

\begin{tabular}{|l|l|l|l|l|l|}
\hline \multicolumn{3}{|c|}{$\begin{array}{l}\text { Number of days to get relief from constipation } \\
\text { after consumption of raisins and CB or ghee }\end{array}$} & \multicolumn{2}{l|}{$\begin{array}{l}95 \% \text { Confidence Interval of the } \\
\text { Difference }\end{array}$} \\
\hline $\mathrm{N}$ & Mean & $\begin{array}{l}\text { Std. } \\
\text { Deviation }\end{array}$ & Std. Error Mean & Lower & Upper \\
\hline 51 & 3.61 & 1.28 & 0.18 & 3.25 & 3.97 \\
\hline
\end{tabular}

The average number of days to get relief from constipation after consumption of raisins and CB or ghee is 3.61 days with standard deviation of 1.28 . The $95 \%$ confidence interval for number of days to get relief from constipation after consumption of raisins and CB or ghee for population is (3.25 days, 3.97 days) approximately ( 3 days, 4 days) that is; In the population the number of days to get relief from constipation after consumption of raisins and CB or ghee would be 3 to 4 days with $95 \%$ confidence.

Thus the study suggested that majority of patients recovered from constipation by taking raisins and $\mathrm{CB}$ or ghee and stopped taking their previous laxatives or enema. All of them got relief within 3-7 days, also got relief from gaseous abdominal distension i.e. bloating, PR bleeding and abdominal pain causes due to constipation. Above findings suggest that there was a significant effect of raisins and $\mathrm{CB}$ or ghee on constipation. In this study not a single patient reported any adverse effect by consuming raisins and $\mathrm{CB}$ or ghee. 


\section{CONCLUSION}

Daily consumption of raisins and clarified butter or ghee for 15 days had given relief from acute and chronic constipation; it also worked on adult as well as pediatric patients. These raisins and clarified butter or ghee act very well on irritable bowel syndrome, fissure in ano and hemorrhoids and helped to stop PR bleeding and bloating causes by these diseases. Only three patients out of fifty-one restarted laxative medicines which they were taking before enrollment in this study, other forty-eight patients did not restart laxatives, this is the major and useful finding of this study. Also all patients got relief from constipation on $3^{\text {rd }}$ and $4^{\text {th }}$ day.

Thus this study concluded that raisins with clarified butter or ghee can be taken instead of laxatives or enema to get relief from constipation; this might be the great substitute for laxatives and per rectum enema.

\section{LIMITATIONS}

Patients of Constipation in pregnancy and menopause were not included in this study.

\section{REFERENCES}

1. Bharucha, A. E., Pemberton, J. H., \& Locke, G. R. (2013). American Gastroenterological Association technical review on constipation. Gastroenterology, 144(1), 218-238.

2. Schiller, L. R. (2001). The therapy of constipation. Alimentary pharmacology \& therapeutics, 15(6), 749-763.

3. American college of gastroenterology. (2004, June). Constipation and defecation problems. Https://Gi.Org/. https://gi.org/topics/constipation-and-defection-problems/

4. Forootan, M., Bagheri, N., \& Darvishi, M. (2018). Chronic constipation: A review of literature. Medicine, 97(20).

5. Constipation. National Digestive Diseases Information Clearinghouse. https://www.niddk.nih.gov/health-information/digestive-diseases/constipation/all-content. Accessed June 20, 2021.

6. Stanford health care. (2017). Constipation. https://Stanfordhealthcare.Org/. https://stanfordhealthcare.org/medical-conditions/primary-care/constipation/complications.html

7. Abbott. (2015, June 16). Press Release $\mid$ Abbott India $\mid$ Abbott shares findings of the 'GutHealth Survey' [Press release]. https://www.abbott.in/media-center/press-releases/abbott-guthealth-survey.html

8. Felman, A. (2019, November). What to know about constipation. https://Www.Medicalnewstoday.Com/. https://www.medicalnewstoday.com/articles/150322

9. Watanabe, T., Nakaya, N., Kurashima, K., Kuriyama, S., Tsubono, Y., \& Tsuji, I. (2004). Constipation, laxative use and risk of colorectal cancer: The Miyagi Cohort Study. European journal of cancer, 40(14), 2109-2115.

10. Vincent, J. L., \& Preiser, J. C. (2015). Getting critical about constipation. Practical Gastroenterology, 144, 14-25 . 
American Journal of Food Sciences and Nutrition

ISSN 2789-3154 (Online)

Vol.3, Issue 2, pp 1- 15, 2021

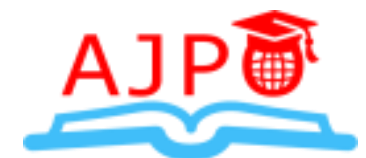

www.ajpojournals.org

11. Karadeniz, F., Durst, R. W., \& Wrolstad, R. E. (2000). Polyphenolic composition of raisins. Journal of Agricultural and Food Chemistry, 48(11), 5343-5350.

12. Camire, M. E., \& Dougherty, M. P. (2003). Raisin dietary fiber composition and in vitro bile acid binding. Journal of agricultural and food chemistry, 51(3), 834-837.

13. Anderson, J. W., \& Waters, A. R. (2013). Raisin consumption by humans: effects on glycemia and insulinemia and cardiovascular risk factors. Journal of food science, 78(s1), A11A17.

14. Kelly, G. (2008). Inulin-type prebiotics--a review: part 1. Alternative Medicine Review, 13(4).

15. Kolida, S., \& Gibson, G. R. (2007). Prebiotic capacity of inulin-type fructans. The Journal of nutrition, 137(11), 2503S-2506S.

16. Anderson, J. W., \& Waters, A. R. (2013). Raisin consumption by humans: effects on glycemia and insulinemia and cardiovascular risk factors. Journal of food science, 78(s1), A11A17.

17. Kim, Y., Hertzler, S. R., Byrne, H. K., \& Mattern, C. O. (2008). Raisins are a low to moderate glycemic index food with a correspondingly low insulin index. Nutrition Research, 28(5), 304-308.

18. Parker, T. L., Wang, X. H., Pazmiño, J., \& Engeseth, N. J. (2007). Antioxidant capacity and phenolic content of grapes, sun-dried raisins, and golden raisins and their effect on ex vivo serum antioxidant capacity. Journal of agricultural and food chemistry, 55(21), 8472-8477.

19. Ghee: Is it good for you? (2020, September 30). Https://Www.Webmd.Com. https://www.webmd.com/diet/ghee-good-for-you\#2-6

20. Sharma, H., Zhang, X., \& Dwivedi, C. (2010). The effect of ghee (Clarified Butter) on serum lipid levels and microsomal lipid peroxidation. Ayu, 31(2), 134-140. https://doi.org/10.4103/0974-8520.72361

21. Kiran, B., Pujitha, P., Sruthi, M., \& Kumar, M. M. (2015). Effect of cow ghee on oral absorption of drugs -An in-vitro comparitive research study

22. Dawson-Hughes, B., Harris, S. S., Lichtenstein, A. H., Dolnikowski, G., Palermo, N. J., \& Rasmussen, H. (2015). Dietary fat increases vitamin D-3 absorption. Journal of the Academy of Nutrition and Dietetics, 115(2), 225-230. https://doi.org/10.1016/j.jand.2014.09.014

23. Lad, V. (1999). The complete book of ayurvedic home remedies. Harmony.

24. Sharma, H. M. (1990). Butter oil (ghee)-Myths and facts. Indian J Clin Pract, 1, 31-2.

25. Ghafoorunissa G. (2008). Role of trans fatty acids in health and challenges to their reduction in Indian foods. Asia Pacific journal of clinical nutrition, 17 Suppl 1, 212-215.

26. Singh, R. B., Niaz, M. A., Ghosh, S., Beegom, R., Rastogi, V., Sharma, J. P., \& Dube, G. K. (1996). Association of trans fatty acids (vegetable ghee) and CB (Indian ghee) intake with higher 
risk of coronary artery disease in rural and urban populations with low fat consumption. International journal of cardiology, 56(3), 289-298.

27. Kumar, A., Tripathi, S., Hans, N., Pattnaik, H. S. N., \& Naik, S. N. (2018). Ghee: Its Properties, Importance and Health Benefits. Lipid Universe, 6, 6-14.

28. Kwak, H. S., Ganesan, P., \& Mijan, A. M. (2013). Butter, ghee, and cream products. Milk and Dairy Products in Human Nutrition: Production, Composition and Health, 390-411.

29. Nebhinani, Mamta \& Saini, Sushma. (2014). Prevalence of Constipation Among the General Population A Community-Based Survey From India. Gastroenterology nursing : the official journal of the Society of Gastroenterology Nurses and Associates. 37. 425-9. 10.1097/SGA.0000000000000074.

30. Vazquez Roque, M., \& Bouras, E. P. (2015). Epidemiology and management of chronic constipation in elderly patients. Clinical interventions in aging, 10, 919-930. https://doi.org/10.2147/CIA.S54304.

31. Loening-Baucke, V., \& Swidsinski, A. (2007). Constipation as cause of acute abdominal pain in children. The Journal of pediatrics, 151(6), 666-669.

32. Dehghani, S. M., Kulouee, N., Honar, N., Imanieh, M. H., Haghighat, M., \& Javaherizadeh, H. (2015). Clinical manifestations among children with chronic functional constipation. Middle East journal of digestive diseases, 7(1), 31.

33. Forootan, M., Bagheri, N., \& Darvishi, M. (2018). Chronic constipation: A review of literature. Medicine, 97(20).

34. Xing, Jin \& Soffer, Edy. (2001). Adverse effects of laxatives. Diseases of the colon and rectum. 44. 1201-9. 10.1007/BF02234645. 